\title{
Safe and Secure Approaches at Lysıs Procedures
}

\section{TO THE EDITOR:}

After intensively reading our article, "Comparison of 3 Approaches to Percutaneous Epidural Adhesiolysis and Neuroplasty in Post Lumbar Surgery Syndrome, by: Akbas, M et al. PAIN PHYSICIAN 2018;21(5)."

I think that those who apply the pain treatments are also interested. I'm sending this "letter to editor" to emphasize our purpose in our article

Racz's minimal invasive epidural catheter procedure, also known as "epidural neuroplasty" is not only utilized in patients suffering from failed back surgery syndrome, but also increasingly applied to nonsurgical back pain patients.

Many studies have proven the efficiency of PEAN - mainly the caudal approach - in managing refractory back/leg pain following PLSS (19-24). Sixty consecutive PLSS patients were recruited and randomized into 3 groups (caudal, S1 foraminal, and L5-S1 transforaminal) before receiving adhesiolysis and neuroplasty.

All procedures were performed under fluoroscopy in a sterile operating room equipped to monitor patients' vital data. A specially designed 16-gauge RX Coude $^{\circledR}$ needle and a Racz ${ }^{\circledR}$-catheter (Epimed International Inc., Johnstown, NY) were used for each of the 3 approaches.

Similarly, our study has shown that, using any of the 3 approaches, PEAN is effective in caudal, S1 foraminal, and L5-S1 transforaminal approaches, evidenced by significant decreases in pain scores. Regarding the incidence of adverse events, our study showed that all of the 3 approaches can be considered safe with low complication rates. There were no statistically significant differences in complication rates between the 3 anatomical approaches. These results are similar to those of previous randomized trials $(27,28)$. Although the $\mathrm{S} 1$ foraminal approach showed a higher number of complications compared to the caudal and L5-S1 transforaminal approaches, this could be attributed to the novelty of the S1 approach and the need for more physician training and practice.

In our study suggests that the 3 anatomical approaches (caudal, S1 foraminal, and L5-S1 transforaminal) used in PEAN have the same pain relief outcomes and complication rates.

In our scientific meetings, our article was very popular and we received positive opinions on this subject. For this reason, we sent this article to draw attention to lisis applications.

The most important factor in the success of these procedures is to perform interventional pain treatment by a specialist who has experience and manage the process by an experienced team. Physcians should choose to perform the lysis procedures with the thecnique which they are good at. so this will increase the safety and reduce the complications also these techniques should be performed in specialized centers by trained physicians.

Mert Akbas, MD, FIPP

Associate Professor

Akdeniz University Medical Faculty

Department of Anesthesiology Division of Algology

07070 - ANTALYA / TURKEY

E-mail: akbasmert@akdeniz.edu.tr 